\title{
Fundamental Design Criteria for Logical Scenarios in Simulation-based Safety Validation of Automated Driving Using Sensor Model Knowledge
}

\author{
Lukas Elster $^{1 *}$, Clemens Linnhoff ${ }^{1 *}$, Philipp Rosenberger ${ }^{1 *}$, Simon Schmidt $^{2 *}$, \\ Rainer Stark $^{3 *}$, and Hermann Winner ${ }^{1 *}$
}

\begin{abstract}
Scenario-based virtual validation of automated driving functions is a promising method to reduce testing effort in real traffic. In this work, a method for deriving scenario design criteria from a sensor modeling point of view is proposed. Using basic sensor technology specific equations as rough but effective boundary conditions, the accessible information for the system under test are determined. Subsequently, initial conditions such as initial poses of dynamic objects are calculated using the derived boundary conditions for designing logical scenarios. Further interest is given on triggers starting movements of objects during scenarios that are not time but object dependent. The approach is demonstrated on the example of the radar equation and first exemplary results by identifying relevance regions are shown.
\end{abstract}

\section{INTRODUCTION}

The release of automated driving functions requires an enormous testing effort. Since this cannot be handled economically in road traffic and on proving grounds, it is increasingly being virtualized [1]-[4]. However, redeployment of testing activities to a virtual vehicle environment opens up the question of appropriate design criteria for test cases.

As e.g. discussed by Neurohr et al. [5], test cases in the virtual vehicle environment are often designed expert- or data-driven by eliciting scenarios on different levels of abstraction (functional, logical, and concrete [6]). For scenariobased testing, the six-layer model (6LM) for scenarios by Scholtes et al. [7] is used to systematically describe scenes and scenarios based on an ontology, as e.g. described by Bagschik et al. [8]. Here, test cases can be designed with elements following the subsequent layers, from road type \& roadway environment, to static \& dynamic objects, to weather conditions, and up to digital infrastructure. The layer model attempts to establish a systematic and uniform way of description for scenes and scenarios.

In addition to the systematization of the test case design, a feasible testing effort is also cited as a motivation [7]. Nevertheless, this cannot be reasoned with the 6LM itself or its application in an ontology based approach, as it structures the testing effort, but does not inherently bring any reduction. Furthermore, a reduction by excluding scenarios raises the question of their reasonability in the first place.

\footnotetext{
${ }^{1}$ The authors are with the Institute of Automotive Engineering, Technical University of Darmstadt, 64287 Darmstadt, Germany $<$ prename $>$. <surname $>$ etu-darmstadt.de

${ }^{2}$ Simon Schmidt is with the Volkswagen AG, Wolfsburg, Germany simon.schmidt 5evolkswagen. de

${ }^{3}$ Rainer Stark is with the Department of Industrial Information Technology, Technische Universität Berlin, Berlin, Germany rainer.stark@tu-berlin.de

*The authors contributed equally to the work by collaboration.
}

For a coherent well-structured safety argumentation, expert-based scenario elicitation should be "executed systematically and supported by automation." [5, p. 125] However, sensor specifications are usually only available for ideal conditions not covering diverse real world circumstances (e.g. weather conditions or different object reflectivities) $[9$, p. 20]. Therefore, it is not possible to extract the concrete boundary conditions for each parameter of a logical scenario only based on the System Under Test (SUT)'s sensor specifications. In other words, fundamental design criteria based on sensor (model) knowledge are needed to prevent useless scenarios to concentrate scenario reduction methods.

\section{RELATED WORK}

Schuldt et al. [10] already propose the formation of equivalence classes, a subsequent boundary analysis, followed by combinatorical test case generation. They derive test cases from their four-layer scenario model, but do not go more into detail on the reasonability of the different scenarios within the immense parameter space that is spanned in such a way. These huge parameter spaces are not possible to be covered [11, p. 539], [12, p. 77f.], even in simulation using a virtual vehicle environment. Taking up the dissertation of Schuldt [13] as the main author of the aforementioned scenario layer model, Amersbach [14] introduces a generic method to facilitate combinatorical test case reduction with $t$-wise testing by functional decomposition into six independent functional layers, as depicted in Fig. 1, derived from the human decision process analysis [15].

Amersbach states that "most of the influence parameters only have an influence on some of the layers, e.g. the majority of the environment representation parameters only affect the functional layers $1 \& 2 "$ [14, p. 88]. All approaches have in common that there is no discussion on the reasonability of test cases regarding the SUT or its Operational Design Domain (ODD). They lack detailed insight into the cause-effect chains within the perception of automated vehicles: Schuldt e.g. completely excludes sensor influence on that matter, stating that "sensor technology is not analyzed further" [13, p. 145].

Since the virtual vehicle environment is mainly perceived via environment sensors, the key for designing reasonable scenarios lies within the functional layers 0 - 2 from [14] covering the perception as input of the higly automated driving function (HADF). Philipp et al. recently published a failure-oriented approach for a deeper look into the perception reasoned by the statement that "the verification of 
Accepted article for the Workshop on Ensuring and Validating Safety for Automated Vehicles

at the 32nd Intelligent Vehicles Symposium (IV), Nagoya, Japan, July 11-15 2021.

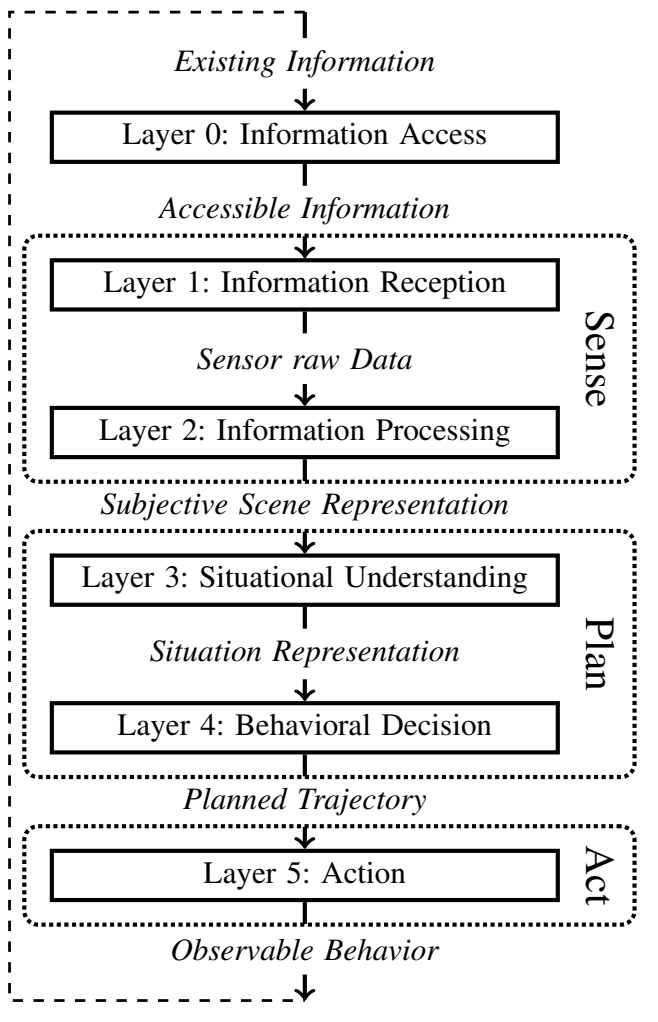

Fig. 1: Functional layers and interfaces as defined by Amersbach [14, p.59], augmented by Philipp et al. [16, p. 4] with the high-level sense-plan-act scheme of Brooks [17]

the perception component is challenging and therefore of special interest" [16, p. 1]. Instead of reasonable test design, as presented in the following, they analyze the perceptual threads in detail, using a tree-based error-failure chain classification scheme. Consequently, the functional layers 3 - 5 from Amersbach [14] will not be discussed in this work, which focuses on the perception layers.

As already mentioned, all information about the virtual vehicle environment is passed to the situational understanding layer by the perception sensors, when disregarding the minor contribution of vehicle communication. Therefore, the key for reasonable test case generation in simulation-based safety validation of automated driving is knowledge about the perception sensor model specification and implementation. In this way, elementary boundary conditions can be identified, such as a geometrically limited field of view of a sensor (model). All information outside this region is not perceived in simulation and therefore does not have to be represented in test cases. This rough filter for sensor boundary conditions is called layer 0 "Information Access" by Amersbach. It comes into play even before the perception starts its own information filter to the driving function within the SUT, as depicted with "sense" by Philipp et al. in Fig. 1 [16]. Within layer 0, globally available ground truth (GT) is reduced to a sensor available GT, while neglecting non-perceivable items for every sensor under the current conditions. It is not limited to a simple range and angle of view from the sensor specification, but also influenced for example by occluding environmental conditions defined in the ODD [18]. Amersbach states: "According to Graab et al. [15] faults on layer 0 could be non-accessible information, obstructed information or masked information due to weather effects (e.g. snow-covered lane markings)".

Therefore, fundamental design criteria for logical scenarios are needed to exclude simulated irrelevant items and / or scenarios from the test suite. This is possible based on the fact that the SUT, the ODD, functional scenarios and especially the sensor system models are already specified and (partly) implemented at the point of test suite design.

The contribution of this work consists of the mentioned fundamental design criteria for logical scenarios. Sensor technology specific equations establish boundaries for the accessible information (layer 0) in simulation. The initial scenario conditions, such as starting poses of the objects, are therefore limited within the derived boundaries for each logical scenario, excluding (parts of) scenarios without sensor model output. Furthermore, triggers are introduced, which start movements of dynamic objects during the scenarios that are not time but distance dependent, using the identified boundaries. The approach is demonstrated on the example of a radar based emergency brake function in a cross-walk scenario and first exemplary results are provided.

\section{From Sensor Model to Logical Scenarios}

To systematically keep the testing effort minimal by design, sensor model boundary conditions are utilized to define the information access layer for the design of logical scenarios with the methodology depicted in Fig. 2. The gray boxes are the contributions of this work and are referenced in the following section in bold letters.

As a prerequisite, the ODD and the SUT are clearly defined and functional scenarios to cover the situational conditions of the ODD are designed for virtual testing. In addition, specifications for the sensor models to be used in the virtual test setup are derived from the boundaries of the ODD and the SUT. With these given conditions, sensor model knowledge is used to exclude distinct parameter values and combinations that are irrelevant to the particular sensor. For example, an object that cannot be detected $200 \mathrm{~m}$ away in rainy conditions is not relevant and therefore does not have to be simulated. There is one exception: Objects that are emitting radiation in the wavelength range of the considered sensor technology. These objects have to be considered separately and are out of scope for this work. Boundaries of parameter value ranges and of specific parameter combinations are set by utilizing the known boundaries of the sensor model defined by its specification and available functional parameters. These boundaries are derived only with the knowledge of the sensor model physics and stochastics parameterized with the parameter values defined in the SUT and the functional scenarios. This allows for an estimation of the maximum perception area of a sensor model, without actually running the simulation. Spacial perception boundaries are for example represented by the sensor range equation. The equation gives the maximum range the sensor 
(model) can receive a signal from an object under given circumstances.

In case of a radar sensor, the maximum range for a given azimuth angle $\phi$ is computed according to [19] as

$$
r_{\max }(\phi)=\sqrt[4]{\frac{10^{-2 \kappa r_{\max }(\phi)} \cdot \sigma \lambda^{2} \cdot G_{\mathrm{Tx}}(\phi) G_{\mathrm{Rx}}(\phi) \cdot P_{\mathrm{Tx}}}{(4 \pi)^{3} \cdot P_{\mathrm{N}} \cdot P_{\Xi}}} .
$$

The maximum range is influenced on one hand by parameters of the deployed sensor itself:

- Transmission power $P_{\mathrm{Tx}}$,

- Antenna diagram for transmission and reception with respect to azimuth angle $G_{\mathrm{Tx}}(\phi) G_{\mathrm{Rx}}(\phi)$,

- Wavelength $\lambda$,

- Receiver noise and signal-to-noise threshold $P_{\mathrm{N}} \cdot P_{\Xi}$, and on the other hand by objects and environmental conditions defined in the scenarios:

- Radar cross section $\sigma$ as a measurement for (instantaneous) reflectivity of a defined object class,

- Attenuation by atmospheric aerosols $\kappa$.

With Eq. (1), which needs to be solved numerically, visibility boundaries for certain objects are defined, parameterized by sensor (setup) properties and scenario environmental conditions. These boundaries form a relevance region for the sensor (setup) defining the maximum detection range for certain objects at certain angles while also considering atmospheric attenuation. An example for such a relevance region of a radar sensor for the pedestrian object class is depicted as a green area in Fig. 3. The object reflectivity $\sigma$ is set to the maximum estimated value of the object's class. For a radar sensor, the reflectivity is described in form of a radar cross section (RCS) given in $\mathrm{dB} \mathrm{m}^{2}$. In literature, the maximum RCS for a pedestrian is estimated at $4.8 \mathrm{~dB} \mathrm{~m}^{2}$ [20]. The attenuation by atmospheric aerosols $\kappa$ represents the influence of environmental conditions like rain, snow, fog etc. on the signal propagation. Since it is highly dependant on the wavelength and polarization of the specific sensor, it is not directly a scenario parameter. It can however be derived e.g. from a given rain rate $R$ in $\mathrm{mm} / \mathrm{h}$ by $\kappa=k R^{\alpha}$ [21]. The parameters $k$ and $\alpha$ depend on the frequency and polarization of the sensor and are selected from look-up tables in [21].

The relevance region, defined with the described parameters, is then enlarged with a safety margin to allow consideration of edge effects and known model stochastics. This relevance region describes the visibility boundaries of a given sensor for a certain object class under defined environmental conditions. By superimposing the visibility boundaries of multiple sensors a unified relevance region for the entire sensor setup is defined. In the example of Fig. 3 the relevance regions of a radar near scan and far scan are combined. This region marks the boundaries of the accessible information and therefore defines layer 0: Information Access on the basis of sensor and scenario knowledge. Every object not contained in the accessible information cannot be detected by the sensor system (model). A test based on such an object will fail and is therefore excluded a priori.

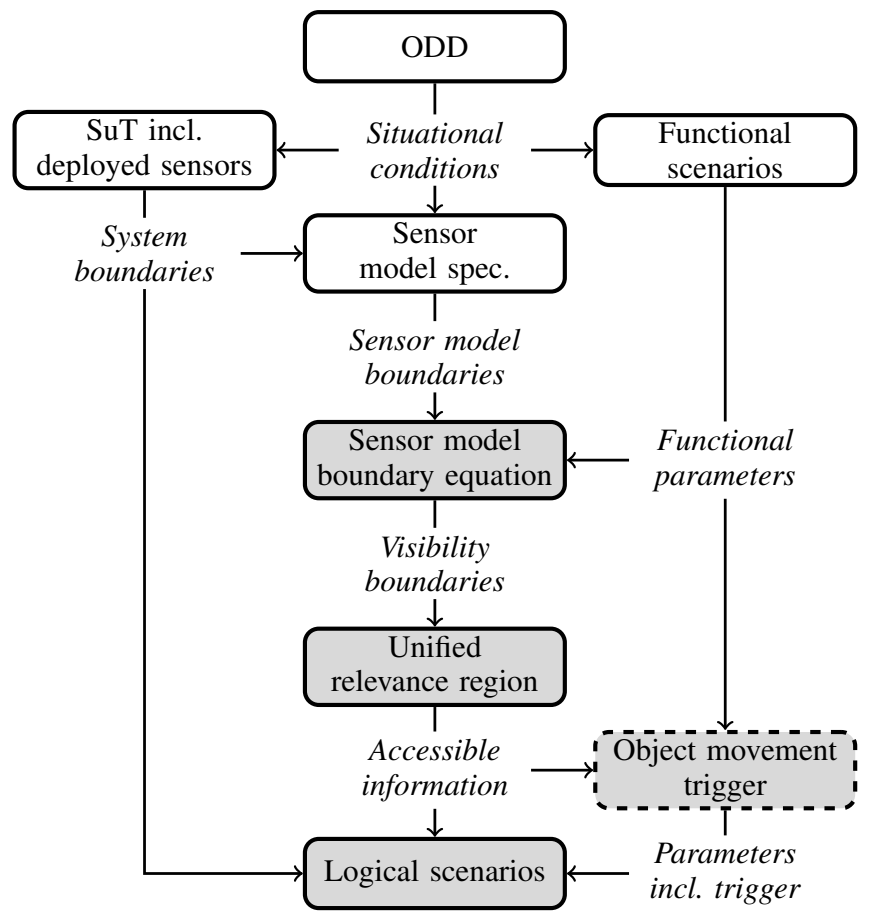

Fig. 2: Proposed method to derive logical scenarios from the Information Access Layer with knowledge from the system under test (SuT) within the designated ODD. The contributions of this work are marked in gray.

While the restriction of the information access already drastically reduces the parameter space for the logical scenarios, the time component leaves room for further reduction, especially for closed-loop testing, where the ego trajectory is not predefined. Only the state of the ego at the beginning of the scenario is defined in form of initial conditions. These conditions are scenario parameters and contain the initial pose and velocity of the ego vehicle. By placing the ego at the start of the scenario in a way that the closest moving object is right at the boundary of the relevance region, the entire region will be covered through the relative movement of the ego vehicle to the object. The initial poses of all moving objects including the ego vehicle are constrained, while all other conditions, like velocities, environmental conditions etc. are still freely variable. The moment, when an object starts moving, is defined by a distance trigger relative to the ego vehicle, marked with dashed lines in Fig. 2. Therefore, movement only takes place in the region that is relevant for the perception sensor setup. The result is a set of logical scenarios with all parameters and parameter combinations within boundaries, that are relevant for the specific SUT.

In conclusion, the design criteria for logical scenarios are twofold. First, the parameter space defining the spacial placement of dynamic objects is set by excluding all parameter combinations outside of the sensors visibility region. Second, distance based triggers are introduced to limit object movement to the identified relevance region. 


\section{Application of the Method}

In this chapter the previously described method is implemented by means of a concrete example. The SUT is an exemplary emergency brake function as a module of a HADF, which is capable of driving in urban areas during dry and rainy weather conditions. The perception sensor setup for the brake function consists of a front radar with a near scan and a far scan in order to implement the unified relevance region of a multi-sensor setup.

To demonstrate the described method a simple functional scenario is chosen. As shown in Fig. 3, the functional scenario is a crosswalk in front of the ego vehicle and a pedestrian with the intention of crossing the street. The coordinate system is a Cartesian system with the $x$-axis in the driving direction of the ego and the $y$-axis to the left with respect to the ego vehicle's driving direction. In this coordinate system the movement of the pedestrian is in positive $y$ direction.

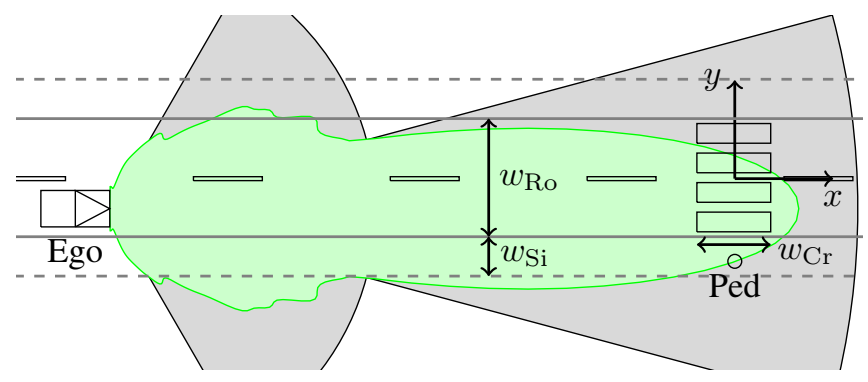

Fig. 3: Functional scenario with unified relevance region

$\square$ : Unified sensor field of view from sensor specs,

$\square$ : Unified relevance region from sensor model knowledge

For the description of the logical scenario parameters, their value boundaries and the discretization are derived (Tab. I). In a first step, the pedestrian position is addressed. The position of the pedestrian depends on the width of the road $w_{\text {Ro }}$, the width of the sidewalk $w_{\mathrm{Si}}$ in $y$ direction and the width of the crosswalk $w_{\mathrm{Cr}}$ in $x$ direction.

Taking the accessible information of the sensor setup into account, the unified relevance region based on the transmitting and receiving antenna diagrams $\left(G_{\mathrm{Tx}}(\phi), G_{\mathrm{Rx}}(\phi)\right)$ of the near and far scan, the RCS of the pedestrian and the attenuation is shown as the green area in Fig. 3. In the proposed approach the position of the pedestrian is calculated by combining the $x_{\mathrm{Ped}}$ and $y_{\mathrm{Ped}}$ position of the pedestrian with respect to the dependency $y_{\text {Ped }}\left(w_{\mathrm{Ro}}\right)$. The possible location of the pedestrian in the scenario is visualized in Fig. 4 by the dark grey area. As an example for this variation two possible initial positions of the pedestrian marked as blue and red dots are shown.

Afterwards, the unified relevance region is utilized to calculate possible relative positions of the ego vehicle to the pedestrian. In this step, equation (1) is solved numerically to calculate $r_{\max }(\phi)$. The mentioned equation depends on the attenuation by environmental conditions, which are represented in the functional scenario as rain with a rain intensity
TABLE I: Parameters and parameter ranges

\begin{tabular}{|c|c|c|}
\hline Parameter & Value range & Discretization \\
\hline Environment condition & & \\
\hline Rain Intensity $I_{\mathrm{Ra}}$ & {$[0,100 \mathrm{~mm} / \mathrm{h}]$} & $5 \mathrm{~mm} / \mathrm{h}$ \\
\hline Scenario parameter & & \\
\hline Road width $w_{\text {Ro }}$ & {$[5.5 \mathrm{~m}, 7.5 \mathrm{~m}]$} & $0.25 \mathrm{~m}$ \\
\hline Sidewalk width $w_{\mathrm{Si}}$ & $2.5 \mathrm{~m}$ & \\
\hline Crosswalk width $w_{\mathrm{Cr}}$ & $4 \mathrm{~m}$ & \\
\hline Ego width $w_{\text {Ego }}$ & $1.7 \mathrm{~m}$ & \\
\hline$v_{x, \text { Ego }}$ & $(0,14 \mathrm{~m} / \mathrm{s}]$ & $0.5 \mathrm{~m} / \mathrm{s}$ \\
\hline Pedestrian position $x_{\mathrm{Ped}}$ & {$[-3 \mathrm{~m}, 3 \mathrm{~m}]$} & $0.5 \mathrm{~m}$ \\
\hline Pedestrian position $y_{\mathrm{Ped}}$ & $\begin{array}{l}{\left[\frac{w_{\mathrm{Ro}}}{2}+w_{\mathrm{Si}}\right.} \\
\left.-\frac{w_{\mathrm{Ro}}}{2}-w_{\mathrm{Si}}\right]\end{array}$ & $0.2 \mathrm{~m}$ \\
\hline $\begin{array}{l}\text { Pedestrian velocity } v_{y, \text { Ped }} \\
\text { RCS } \sigma_{\text {Ped }}\end{array}$ & $\begin{array}{l}(0,4 \mathrm{~m} / \mathrm{s}] \\
4.8 \mathrm{~dB} \mathrm{~m}^{2}\end{array}$ & $0.2 \mathrm{~m} / \mathrm{s}$ \\
\hline Sensor parameter & & \\
\hline Transmitted power $P_{\mathrm{Tx}}$ & $10 \cdot 10^{-3} \mathrm{~W}$ & \\
\hline Transm. antenna diagram $G_{\mathrm{Tx}}(\phi)$ & $\ldots$ & \\
\hline Receiver antenna diagram $G_{\mathrm{Rx}}(\phi)$ & $\ldots$ & \\
\hline Wavelength $\lambda$ & $3.92 \cdot 10^{-3} \mathrm{~m}$ & \\
\hline Detection min. power $P_{\mathrm{N}} \cdot P_{\Xi}$ & $2.58 \cdot 10^{-15} \mathrm{~W}$ & \\
\hline Azimuth resolution $\Delta \phi$ & $1.6^{\circ}$ & \\
\hline Range resolution $\Delta r$ & $1.5 \mathrm{~m}$ & \\
\hline Trigger & & \\
\hline Relative position trigger $T_{\mathrm{Ego}, \mathrm{Ped}}$ & $\begin{array}{l}{\left[\min \left(r_{\max }\right)\right.} \\
\left.\max \left(r_{\max }\right)\right]\end{array}$ & $0.5 \cdot \Delta r$ \\
\hline
\end{tabular}

$I_{\mathrm{Ra}}$. The angles of the antenna diagram are discretized at half the sensor azimuth resolution $\Delta \phi$ to ensure getting at least one sample per azimuth bin. As a result, all possible discrete initial positions of the ego vehicle $\left(x_{\mathrm{Ego}}, y_{\mathrm{Ego}}\right)$ based on the unified relevance region are known. Fig. 4 shows additionally to the two different possible variations of the pedestrian's initial position the unified relevance region of the sensor as blue and red solid, dashed and dotted line. The iteration process for three different discrete angles of the relevance region is shown in Fig. 4a and Fig. 4b with the corresponding ego vehicle position $\mathrm{Ego}_{1 / 2,1 / 2 / 3}$ based on a fixed initial pedestrian position $\operatorname{Ped}_{1 / 2}$. The position of the $\mathrm{EgO}_{2,3}$ seems to be a start position, that has no relevance because of the pedestrian's moving direction. But for closed loop simulations this position could be especially challenging for the SUT.

The described calculation is done with respect to boundary conditions, which are the defined width of the vehicle $\left(w_{\text {Ego }}\right)$, the road width and the prerequisite, that the ego vehicle should be in the right lane of the road. Therefore, only realistic positions of the pedestrian and the ego vehicle as start conditions for the scenario remain.

In a last step, the newly introduced trigger parameter $T_{\text {Ego,Ped }}$ is defined. This parameter starts the movement of the pedestrian alongside the crosswalk with respect to the decreasing distance between the ego vehicle and the pedestrian. In this example the discrete points are half of the range resolution $\Delta r$ of the radar sensor. Therefore the pedestrian will only move, when he is located in the relevant region of the SUT. This concludes the design of the logical scenarios utilizing boundary conditions from sensor system model knowledge and distance based moving object trigger. 


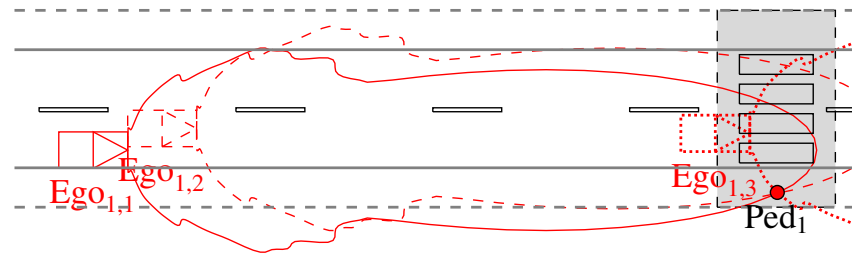

(a) Variation of ego start using relevance regions for $\mathrm{Ped}_{1}$ $i_{-}$Region of possible initial pedestrian positions (-) Start position $\mathrm{Ego}_{1,1}$ with relevance region (- - ) Start position $\mathrm{Ego}_{1,2}$ with relevance region (……..) Start position Ego $_{1,3}$ with relevance region

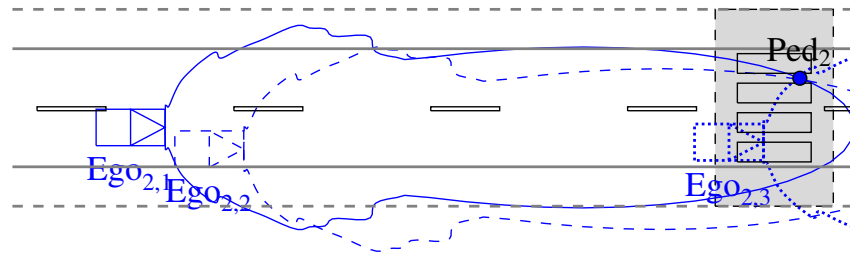

(b) Variation of of ego start using relevance regions for $\mathrm{Ped}_{2}$ i_ Region of possible initial pedestrian positions (-) Start position $\mathrm{Ego}_{2,1}$ with relevance region (- - ) Start position $\mathrm{Ego}_{2,2}$ with relevance region (….....) Start position $\mathrm{EgO}_{2,3}$ with relevance region

Fig. 4: Crosswalk scenario with different relevance regions and start positions for the pedestrian

To compare the knowledge driven approach with an approach combining all position related parameters covered by the field of view from a sensor spec. with an $N$-wise testing approach, the total number of resulting concrete scenarios is calculated. The typical logical scenario based on the sensor data sheet would be created by defining additional parameters for the initial ego position. The longitudinal $x_{\text {Ego }}$ position of the ego vehicle is defined by a high level sensor specification, which gives the maximum range $r_{\max }$ and the maximum field of view (FOV) from a sensor data sheets. This can be seen in Fig. 3 as gray area. In general, the lateral ego position depends on the ego's width $w_{\text {Ego }}$, the road width $w_{\text {Ro }}$ and a margin for the distance to the road boundary of $0.2 \mathrm{~m}$. The number of all unique parameter combinations of the positions for the data sheet driven approach is 7,413,705. In case of the sensor knowledge driven method with the newly introduced trigger parameter the number of possible positions is 24,531. This example points out the importance of integrating sensor knowledge into the definition of logical scenarios.

\section{CONCLUSION AND OUtLOOK}

A method to design reasonable scenarios by using sensor model knowledge was introduced. The method can be used for all perception sensor principles and has to be adapted according to the sensor boundaries. Additionally, the method is applicable to sensor setups consisting of various numbers of sensors and sensor principles by building the intersection of all visibility boundaries. Using the trigger, which depends on relative positions of objects, the parameter space is covered as a naive combination of all positions would do, but less parameter combinations have to be calculated.

The benefit of considering sensor (model) knowledge in the definition process of logical scenarios was shown, as can be seen in Fig. 3. The sensor model generates detections of the pedestrian only if the object is located or moving within the green area. Hence, simulating scenarios or parts of scenarios, where nothing happens within the relevance region has no influence on the SUT at all and no further comprehension of the HADF is generated. Nevertheless, the key point of the method is to calculate the relevance region for every logical scenario to only generate reasonable scenarios in the first place. Therefore, deep knowledge about the sensor principle(s) and signal processing steps is crucial to only consider justified parameter combinations.

The scenario based test effort could be further reduced by subdividing the presented relevance region into parts most important to the SUT. Considering criticality analysis, not all parts of the relevance region might be equally important for the safety validation process. Entering of an object into the relevance region is challenging and an object approaching the ego vehicle becomes increasingly critical. These more important parts of the relevance region should be discretized in smaller steps than the rest of the visibility region or might be left out completely.

When a combined sensor setup is used (common in HADF) different sensor relevance regions are combined. Each depends on several causes and effects, which raises the question of a systematic collection and ontology for such sensor knowledge. Neurohr et al. also point out the need for "an identification of all relevant phenomena." [5, p. 125] Here, the recently started initiative called Perception Sensor Collaborative Effect and Cause Tree (PerCollECT) of the authors could be an option. It is publicly available at https://github.com/PerColleCt.

There, a collaborative approach is proposed to collect the sensor knowledge within the community and to provide it in a tree-based ontology. Key points in this collection are the ordering onto functional layers of the perception sensor system and the required references for each effect and cause within the cause-effect chains and for each connection between them. The authors see the chance to use such an ontology, when the actual state of community knowledge is reached e.g. for ODD design, for coverage analysis when ODD, SUT and the logical scenarios are already determined, or even to design a sensor setup that considers a pre-defined ODD. Based on this ontology functional scenarios for investigation of sensor effects could be derived. The combination of these effects can reduce the number of functional scenarios. For example at a tunnel's entrance the camera and the lidar recognize a change in brightness, multi path propagation of the radar's electromagnetic waves take place and the GNSS sensor loses its signal. 


\section{ACKNOWLEDGMENTS}

This work received funding from SET Level and VVM of the PEGASUS project family, promoted by the German Federal Ministry for Economic Affairs and Energy based on a decision of the Deutsche Bundestag.

The authors would like to thank Christian Amersbach and Ken Thaddäus Mori for the valuable discussions.

This work was presented at the 4th Workshop on Ensuring and Validating Safety for Automated Vehicles (WS13), IV2021

\section{REFERENCES}

[1] J. Zhou, R. Schmied, A. Sandalek, H. Kokal, and L. del Re, "A Framework for Virtual Testing of ADAS," SAE International Journal of Passenger Cars - Electronic and Electrical Systems, vol. 9, no. 1, pp. 66-73, Apr. 2016. [Online]. Available: https://www.sae.org/content/2016-01-0049/

[2] R. Lattarulo, J. Pérez, and M. Dendaluce, "A complete framework for developing and testing automated driving controllers," IFACPapersOnLine, vol. 50, no. 1, pp. 258-263, Jul. 2017. [Online]. Available: https://linkinghub.elsevier.com/retrieve/pii/S2405896317300587

[3] D. Nalic, A. Pandurevic, A. Eichberger, and B. Rogic, "Design and Implementation of a Co-Simulation Framework for Testing of Automated Driving Systems," Sustainability, vol. 12, no. 24, p. 10476, Dec. 2020. [Online]. Available: https://www.mdpi.com/ 2071-1050/12/24/10476

[4] S. Hallerbach, Y. Xia, U. Eberle, and F. Köster, "Simulation-Based Identification of Critical Scenarios for Cooperative and Automated Vehicles," SAE Technical Papers, vol. 2018-01-1066, Apr. 2018.

[5] C. Neurohr, L. Westhofen, T. Henning, T. de Graaff, E. Möhlmann, and E. Böde, "Fundamental Considerations around Scenario-Based Testing for Automated Driving," in 2020 IEEE Intelligent Vehicles Symposium (IV), Oct. 2020, pp. 121-127, iSSN: 2642-7214.

[6] T. Menzel, G. Bagschik, and M. Maurer, "Scenarios for Development, Test and Validation of Automated Vehicles," in 2018 IEEE Intelligent Vehicles Symposium (IV). Changshu: IEEE, Jun. 2018, pp. 1821-1827. [Online]. Available: https://ieeexplore.iee.org/document/ $8500406 /$

[7] M. Scholtes, L. Westhofen, L. R. Turner, K. Lotto, M. Schuldes, H. Weber, N. Wagener, C. Neurohr, M. Bollmann, F. Körtke, J. Hiller, M. Hoss, and L. Eckstein, "6-Layer Model for a Structured Description and Categorization of Urban Traffic and Environment," IEEE Access, p. 16, 2021.

[8] G. Bagschik, T. Menzel, and M. Maurer, "Ontology based Scene Creation for the Development of Automated Vehicles," in 2018 IEEE Intelligent Vehicles Symposium (IV 2018), Apr. 2018. [Online]. Available: https://doi.org/10.1109/IVS.2018.8500632

[9] S. Muckenhuber, H. Holzer, and Z. Bockaj, "Automotive Lidar Modelling Approach Based on Material Properties and Lidar Capabilities," Sensors, vol. 20, no. 11, p. 3309, Jan. 2020, number: 11 Publisher: Multidisciplinary Digital Publishing Institute. [Online]. Available: https://www.mdpi.com/1424-8220/20/11/3309

[10] F. Schuldt, F. Saust, B. Lichte, M. Maurer, and S. Scholz, "Effiziente systematische Testgenerierung für Fahrerassistenzsysteme in virtuellen Umgebungen," in Beiträge zum gleichnamigen 14. Braunschweiger Symposium am Forschungsflughafen, Braunschweig. ITS mobility e.V., 2013.

[11] I. Sommerville, Software Engineering, 8th ed. New York, NY, USA: Addison-Wesley, 2006.

[12] J. Gericke and M. Wiemann, "Optimierte Fehlerfindung im Funktionstest durch automatisierte Analyse von Testprotokollen," in Software Engineering 2007 - Beiträge zu den Workshops - Fachtagung des GI-Fachbereichs Softwaretechnik, S. Böttinger, L. Theuvsen, S. Rank, and M. Morgenstern, Eds. Bonn: Gesellschaft für Informatik e. V., 2007, pp. 75-84, accepted: 2019-05-15T09:38:03Z ISSN: 16175468. [Online]. Available: http://dl.gi.de/handle/20.500.12116/22746

[13] F. Schuldt, "Ein Beitrag für den methodischen Test von automatisierten Fahrfunktionen mit Hilfe von virtuellen Umgebungen," PhD Thesis, Technischen Universität Braunschweig, Braunschweig, Apr. 2017. [Online]. Available: https://publikationsserver.tu-braunschweig. de/servlets/MCRFileNodeServlet/dbbs_derivate_00043536/Diss_ Schuldt_Fabian.pdf
[14] C. T. Amersbach, "Functional Decomposition Approach - Reducing the Safety Validation Effort for Highly Automated Driving," PhD Thesis, TU Darmstadt, 2020. [Online]. Available: https: //tuprints.ulb.tu-darmstadt.de/id/eprint/11520

[15] B. Graab, E. Donner, U. Chiellino, and M. Jokela, "Analyse von Verkehrsunfällen hinsichtlich unterschiedlicher Fahrerpopulationen und daraus ableitbarer Ergebnisse für die Entwicklung adaptiver Fahrerassistenzsysteme," in Active Safety Through Driver Assistance. München: TU München \& TÜV Süd Akademie GmbH, 2008. [Online]. Available: https://mediatum.ub.tum.de/doc/1145118/

[16] R. Philipp, F. Schuldt, and F. Howar, "Functional Decomposition of Automated Driving Systems for the Classification and Evaluation of Perceptual Threats," in 13. Uni-DAS e.V. Workshop Fahrerassistenz und automatisiertes Fahren 2020, Walting, 2020. [Online]. Available: https://www.researchgate.net/publication/343136326_ Functional_Decomposition_of_Automated_Driving_Systems_for_ the_Classification_and_Evaluation_of_Perceptual_Threats

[17] R. Brooks, "A robust layered control system for a mobile robot," IEEE Journal on Robotics and Automation, vol. 2, no. 1, pp. 14-23, Mar. 1986, conference Name: IEEE Journal on Robotics and Automation.

[18] P. 1883:2020, "Operational Design Domain (ODD) taxonomy for an automated driving system (ADS) - Specification," British Standards Institution, Tech. Rep., Aug. 2020. [Online]. Available: https: //www.bsigroup.com/globalassets/localfiles/en-gb/cav/pas1883.pdf

[19] H. Winner, "Automotive RADAR," in Handbook of Driver Assistance Systems: Basic Information, Components and Systems for Active Safety and Comfort, H. Winner, S. Hakuli, F. Lotz, and C. Singer, Eds. Cham: Springer International Publishing, 2016, pp. 325-403. [Online]. Available: https://doi.org/10.1007/978-3-319-12352-3_17

[20] P. Lemmen, J. Stoll, U. Bergelt, P. Seiniger, M. Wisch, O. Bartels, E. Schubert, M. Kunert, I. Knight, D. Brookes, M. Ranovona, T. Okawa, C. Domsch, and T. Schaller, "Evaluation of Pedestrian Targets for Use in Autonomous Emergency Brake System Testing - A Report from the Harmonisation Platform 2 Dealing with Test Equipment," in 23rd Enhanced Safety of Vehicles Conference (ESV). Seoul, Republic of Korea: Euro NCAP, Mar. 2013.

[21] ITU-R, "RECOMMENDATION ITU-R P.838-3 - Specific attenuation model for rain for use in prediction methods," International Telecommunication Union, ITU-R P.838-3, Mar. 2005. 